Our findings were as follows. There was no significant difference in mean blood glucose concentrations between babies in groups $A$ and $B$ on day 0 . There was no significant difference between the two groups in maternal total proteins, nor in any of the serum protein components. The correlation coefficient between maternal total protein levels, albumin, and globulin components in the mothers in groups $A$ and $B$, and blood glucose in their infants showed no significant difference. The correlation coefficient between maternal serum albumin in group $\mathrm{A}$ and the different categories of babies in the same group (i.e. low birthweight, preterm, and small-for-gestational-age) showed no significant difference. Our findings thus differ from those of Stein, though we are dealing with the same type of population.

M. A. PARENT

Department of Paediatrics, Children's Hospital,

P.O. Box 977, Durban, South Africa.

REFERENCES

Powell, S. J. (1958). The serum protein pattern of liver function tests in the Natal African. South African Fournal of Laboratory and Clinical Medicine, 4, 273.

Stein, H. (1975). Maternal protein depletion and small-for-gestational age babies. Archives of Disease in Childhood, 50, 146.

Dr. H. Stein comments as follows:

I read with great interest the letter of Dr. Parent. There are marked differences between his investigations and ours (Archives, 1975). He has principally investigated the contrast in terms of hypoglycaemia and maternal serum protein levels between term infants (group B) and a group of preterm, low-birthweight, and small-for-gestational-age infants (group A). Even in Group A where he contrasts his 3 categories there is only a relatively small percentage of low-birthweight infants (27 out of 99) and it is not clear whether these are preterm or small-for-gestational-age.

We, on the other hand, investigated a group of low-birthweight infants only, but excluded twins, as we had previously shown that these babies contributed significantly to the high incidence of low-birthweight infants seen by us $(26 \%)$ and especially the small-forgestational-age group (Stein and Ellis, 1974); in the present investigation we were looking for additional aetiological factors. Almost all of our cases were preterm (94 out of 103) and the majority were small-forgestational-age.

It appears that the whole emphasis of the investigations was different. It is also not clear whether Dr. Parent excluded twins as we did. Thus while the population groups are similar, the investigations do not appear comparable.

H. STEIN

Department of Paediatrics, Baragwanath Hospital, P.O. Bertsham 2013, Johannesburg, South Africa. REFERENCE

Stein, H., and Ellis, U. (1974). The low birthweight African baby. Archives of Disease in Childhood, 49, 156.

\section{Opsonic defects}

Sir,

The recently described familial opsonic defect by Scott et al. (1975) in the Archives is a beautifully performed study which deserves recognition. The authors have clearly shown a defect in serum opsonic activity throughout four generations of a family. Their suggestion, however, that, 'A diagnosis of Miller's opsonizing defect was confirmed by demonstration of defective opsonization for yeast phagocytosis by her serum and that of her relatives', is a premise with which I do not fully agree.

Most, if not all, opsonic assays depend upon a multiplicity of plasma factors. While Baker's yeast particles are unique in their dependency upon the fifth component of complement (C5) (Miller and Nilsson, 1974), C5 alone is not sufficient for complete opsonization of yeast. In other words, a deficiency in the yeast opsonic assay may reflect any one of a number of factors, including C5. In order to establish a diagnosis of $\mathrm{C} 5$ dysfunction it is therefore necessary to perform appropriate reconstitution experiments using purified human C5 (Miller and Koblenzer, 1972). Illustration of this point is provided by the observations that patients with other opsonic defects, as for example the patient of Alper and Rosen (1975) with hypercatabolism of C3, have abnormal yeast opsonic assays.

I agree that the nature of any defect revealed, solely, by abnormal yeast opsonic activity requires further definition. In the family reported by us, however, substantial evidence has been presented which points to a functional defect of the $\mathrm{C} 5$ molecule as the cause of the opsonic defect (Nilsson, Miller, and Wyman, 1974). The alternate pathway of complement in this family is normal.

The importance of studies such as that of Scott and co-workers is re-emphasized. A number of opsonic defects obviously exist and the full characterization of each should be vigorously pursued. Not only do we learn much of the normal inflammatory response from each of these defects, but the promise of specific therapy by improved characterization of each is great.

MICHAEL E. MILLER Department of Paediatrics, Charles R. Drew Postgraduate Medical School and U.C.L.A. School of Medicine, 1621 E. 120th Street, Los Angeles, California 90059

REFERENCES

Alper, C. A., and Rosen, F. S. (1975). Increased susceptibility to infection in patients with defects affecting C3. Immuno deficiency in Man and Animals, p. 302 . Ed. by R. A. Good and J. Finstad. Sinauer Associates, Sunderland, Mass.

Miller, M. E., and Koblenzer, P. J. (1972). Leiner's disease and deficiency of C5. Fournal of Pediatrics, 80, 879.

Miller, M. E., and Nilsson, U. R. (1974). A major role of the fifth component of complement (C5) in the opsonization of yeast particles. Partial dichotomy of function and immunochemical measurement. Clinical Immunology and Immunopathology, 2 , 246. 
Nilsson, U. R., Miller, M. E., and Wyman, S. (1974). A functional abnormality of the fifth component of complement (C5) from human serum of individuals with a familial opsonic defect. fournal of Immunology, 112, 1164.

Scott, H., Moynahan, E. J., Risdon, R. A., Harvey, B. A. M., and Soothill, J. F. (1975). Familial opsonization defect associated with fatal infantile dermatitis, infections, and histiocytosis. Archives of Disease in Childhood, 50, 311.

\section{Bronchial lability in cystic fibrosis}

Sir,

Counahan and Mearns (1975) report an abnormal degree of bronchial lability in patients with cystic fibrosis, a finding which was also reported by Day and Mearns in 1973. In order to measure lability they describe two alternative indices. The Exercise Lability Index (ELI) which is the rise in PEF plus the fall in PEF expressed as a percentage of the value at rest. Secondly, a modification of the Jones Lability Index (JLI) which is the highest PEF (exercise only) minus lowest PEF, expressed as a percentage of the predicted normal PEF, the modification being omission of a bronchodilator agent to reinforce the bronchodilator effect of brief exercise in order to obtain the highest PEF.

It has always seemed to me that the use of the ELI distorts results and may be misleading because a falsely high value for lability is obtained when the PEF at rest is low. It is incorrect to compare percentage values from such patients with percentage values from those with figures at rest which are near to normal.
The JLI avoids this distortion (Jones, 1966). Counahan and Mearns found in CF that the JLI was normal at $18 \%$, whereas the ELI was abnormal at $24 \%$. Day and Mearns (1973) got into a similar difficulty for the same reason. I do not suggest that a proportion of CF patients do not have abnormal lability but would maintain that the ELI introduces inaccuracy which may blur the distinction between CF and other respiratory conditions and may lead to the postulation of problems concerning lability in CF which do not necessarily exist.

Failure to use a bronchodilator agent to reinforce brief exercise may also cause distortion but in the opposite direction, i.e. it gives a lower figure for lability and one is left uncertain to what extent a low PEF at rest is due to a labile as opposed to a fixed increase of airway resistance.

While these points do not invalidate this valuable and interesting piece of work, they do influence conclusions. Much confusion has arisen in published reports on this subject due to the reporting of experimental data from exercise tests which were technically at fault.

R. S. JONES

Alder Hey Children's Hospital, Eaton Road, Liverpool L12 $2 A P$.

REFERENCES

Counahan, R., and Mearns, M. B. (1975). Prevalence of atopy and exercise-induced bronchial lability in relatives of patients with cystic fibrosis. Archives of Disease in Childhood, 50, 477.

Day, G., and Mearns, M. B. (1973). Bronchial lability in cystic fibrosis. Archives of Disease in Childhood, 48, 355.

Jones, R. S. (1966). Assessment of respiratory function in the asthmatic child. British Medical fournal, 2, 972. 